\title{
PERIPROSTHETIC FRACTURE TREATED WITH A SIMPLE TECHNIQUE: A CASE REPORT
}

Bejoy E Jayan¹, Varunjikar M D², Dnyaneshwar Kale³, Suraj S Mahadik ${ }^{4}$

\section{HOW TO CITE THIS ARTICLE:}

Bejoy E Jayan, Varunjikar MD, Dnyaneshwar Kale, Suraj S Mahadik. "Periprosthetic fracture treated with a simple technique: a case report". Journal of Evolution of Medical and Dental Sciences 2013; Vol. 2, Issue 46, November 18; Page: 8989-8995.

ABSTRACT: The advent and increasing use of joint replacement for various conditions has led to a new group of fractures- the periprosthetic fractures. The hip joint is commonly involved in this group. The management of these fractures is complex and usually needs application of basic principles to fit an individual situation rather than having a fixed set of rules. Standard treatment protocol includes use of locking plate with encirclage wires.

In General Hospital, health and economic problems of poor patients warrants a need for alternative simple technique for management of such cases.

In this article we discuss a case of peri-prosthetic fracture of shaft of femur in a lean, short statured lady, reluctant for blood transfusion due to previous reaction secondary to minor incompatibility, treated by use of ender nail.

CASE REPORT: 55 year female, lean and short statured lady, poorly nourished, from rural area, was brought to OPD with alleged history of fall about a day back with complaint of pain in left thigh and inability to bear weight and deformity. She underwent hemiarthroplasty (Austin moore prosthesis) for fracture neck femur on same side 2 years back.

On examination, there was sub-trochanteric shortening of left lower limb with externally rotation, tenderness present over the junction of upper and middle third of thigh with swelling. Abnormal and painful mobility was elicited at that site.

$\mathrm{X}$-ray of pelvis including both hip joint and left thigh revealed peri-prosthetic fracture femur.

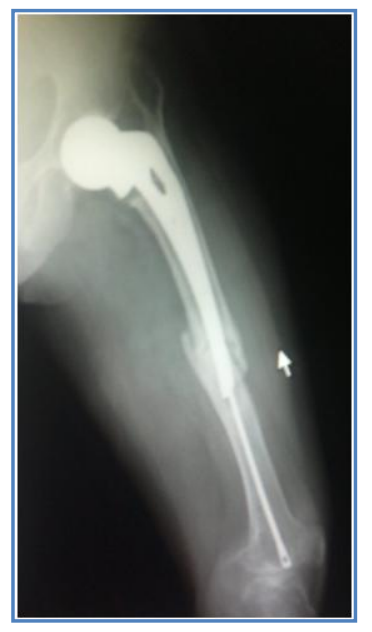

Intra-operative x-ray of peri-prosthetic fracture of left femur

Blood investigations revealed $\mathrm{Hb}$ 7.1. Other relevant investigations especially LFT, RFT were within normal limits. Due to respiratory and cardiac status, she was declared unfit for anaesthesia 
and major surgical procedure. Due to previous history of reaction to blood transfusion, patient was unwilling for the same.

Standard treatment of such fractures is lateral locking plate fixation with encirclage wire.

In this case, due to health, financial constrain, and anticipated blood loss and unwillingness for blood transfusion, standard treatment protocol was not possible.

Considering these factors, patient was initially put on skeletal traction for 3 weeks followed by ender nailing through distal femur under local anaesthesia and sedation. We were planning to fix the fracture with 2 ender nails, but, we could negotiate only one beyond the fracture site. Intraoperatively, stability at fracture site was fair. Post operatively, patient was kept in hospital for 15 days and was advised toe touch weight bearing for 3 months along with quadriceps exercises on discharge. She was followed up regularly every month and assessed clinically and radiologically. Partial / full weight bearing was started after 3 months, and now on follow up of 1 year, patient is able to walk and perform activities of daily routine. To compensate for shortening, patient was advised shoe raise and walking aid.

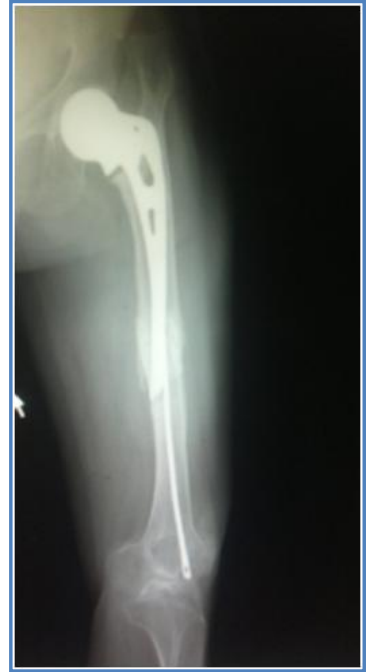

3 months follow-up of periprosthetic fracture left femur and ender nail in-situ

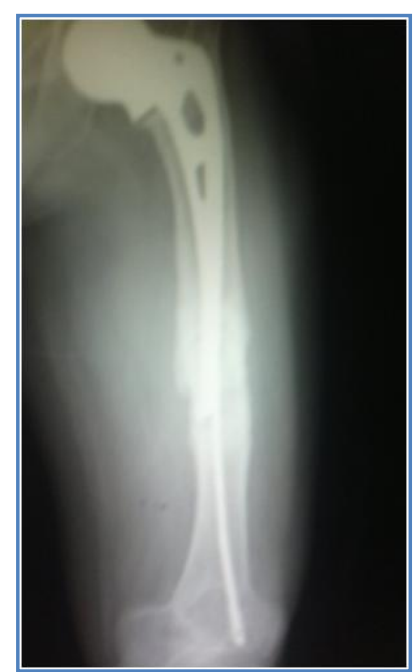

1 year follow-up of periprosthetic fracture left femur with ender nail in-situ 
DISCUSSION: Among the various mode of treatment of long bone fractures, internal fixation has become the mainstay of treatment with emphasis largely on intermedullary fixation. Reports in literature indicating adverse effects of reaming like increase incidence of pulmonary complications and disruption of vascular supply of inner two-thirds of cortex have resulted in increased popularity of undreamed nails for fixation of such fracture. $(1,2)$

Flexible unreamed intramedullary nails have long been used to manage diaphyseal fracture of long bones. These nails rely on three point fixation in the medullary canal and provide favorable condition, as the forces are evenly distributed along entire length of nails. $(7,8)$. As the fixation by these nails is not rigid, therefore some amount of micro motion occurs between the two fragments which in turn stimulates fracture healing. This nails do not ensure sufficient longitudinal stability in grossly comminuted or long oblique fracture with result shortening $(6,9)$.

Manual traction was found to be equally effective and safe with additional advantage of avoiding complications like femoral, pudendal, sciatic nerve injury, pressure sore in the groin region etc. reported in literature with the use of fracture table. (12).

Operative time in ender nailing was much shorter than for $\mathrm{k}$ nailing and interlock nailing of femur. $(4,5)$. The procedure can be done without major blood loss, thus obviating the need for blood transfusion and its possible complications. Average blood in reamed nailing of femur with or without locking has been reported to be $400-2100 \mathrm{ml}(5,12,14)$. Image intensifier exposure timing in present study ranged from 1.5 to 2.5 minutes. After passage of one nail from either condyle, not much of exposure was required for subsequent nails. In reamed locking nailing of femur, average exposure time has been reported to be ranging from 2-20 minutes $(3,16,17,18)$.

Pankovich et. Alon on the other hand have used 2 nails in most of their cases and only 1 nail in 2 of their patients. However, 16 of their cases required post operative cast brace or traction. (6). Higher number of nails provide better stability because of better nail- cortical contact beside decreasing chances of stress fracture of the nails. Moreover, using more number of nailing with both medial and lateral portals ensure better rotational and angular stabilization of the fracture, thereby, permitting early weight bearing. $(9,11)$.

As ender nails are flexible, weight bearing leads to axial micromotion at the fracture site thereby stimulating the internal callus formation. (6) and early partial weight bearing by 1-4 weeks can be allowed without any complication of bending or breaking of nails. $(6,9,11)$. However it is desirable to protect this fracture from weight bearing if number of nails used is two or less or fracture line is unstable as it may lead to angulation. (10).

Closed intramedullary fixation of using ender's nails therefore appears to be a simple, safe and effective method of treating femoral diaphyseal fracture relatively stable configuration. However the method has its limitations in that it cannot be used in unstable comminuted fracture on account of its relatively longitudinal stability.

Hemi- arthroplasty such as AMP is not a product of twenty first century. It was first developed by Gluck in Germany in 1890. He performed a hip replacement with ivory femoral and acetabular, components cemented to bone by a combination of resin and pumice or plaster-of-Paris (22). It rose to popularity late in the twentieth century and has thereafter gained increasing popularity for a number of indications such as in inflammatory, degenerative and traumatic conditions of the hip. The increased use of hip replacement has heralded the onset of a new subset of 


\section{CASE REPORT}

fractures- the periprosthetic fractures (23). The increasing life span \& incidence of osteoporosis contributed to this type of injuries.

Hemiarthroplasty works on the interface between bone-prosthesis, to achieve a mechanical restoration of function of the replaced part. Unfortunately this construct is not entirely inert, and is subject to wear secondary to a number of factors, mainly the hoop stresses at the bone prosthesis interface. This wear phenomenon leads to loosening of the implant with resultant hip pain and occasionally a fracture. The prosthesis placement is well known to create a stress riser effect, which weakens the bone around the region. These factors, combined with the fact that most patients undergoing hip replacement are elderly and osteoporotic leads to the perfect mix for a fracture recipe.

Periprosthetic fractures are thus a well recognized and accepted complication of hemiarthroplasty. The development of treatment of periprosthetic fractures around a hip replacement has come a long way from the initial conservative approach of traction, casting and bed rest. The difficulty, however, in surgical management of what would otherwise appear to be a relatively common complication of a commonly performed surgery is well illustrated by the number of classifications that have arisen to describe these fractures(24-30). Of these, the Vancouver classification (described below) is the most accepted and used. According to the Vancouver classification, periprosthetic fracture treatment decisions depend on five important factors: fracture location, stability of the implant and fracture, quality of host bone stock, patient physiology and age, and surgeon's experience (31).

Fixation may be achieved by several means. Fractures with loose stems require revision arthroplasty with a long stem prosthesis.Fractures with a well fixed stem need fracture fixation which may be accomplished by: a lateral plate with proximal and distal cables (the Dall-Miles system(32); a lateral plate with proximal circlage wires and distal bicortical screws(the Ogden plate(33); plating with proximal unicortical screws and distal bicortical screws; plating with proximal unicortical screws combined with encirclage and distal bicortical screws; and two allograft cortical strut grafts with cables.

In a biomechanical study by Dennis et al(34), it was found that the system of plating with distal bicortical and proximal unicortical screws with or without a cable was biomechanically the most superior of the five systems studied.In another biomechanical study, Lever et al (35) compared the various fixation systems and found that the use of proximal unicortical and distal bicortical screws offered better stiffness and axial stability than use of cables in any instance.

The use of cement- in- cement technique has been studied in the treatment of these fractures. It is felt that this technique helps augment the fracture fixation by providing a further rigid scaffold (36). The hook plate has been used in the treatment of subtrochanteric fractures related to periprosthetic fractures (37).

The algorithm shows Vancouver classification of post op periprosthetic fractures and this preferred treatment:

Type A

A-L: Lesser trochanter,symptomatic treatment only even if displaced. Intervene only if substantial segment medial cortex attached. 
A-G: Greater trochanter, symptomatic treatment with crutches and limit adduction. Intervene only if displaced to avoid pain, weakness, limp or instability

\title{
Type B
}

\author{
Fixation of stem: \\ Well fixed stem -B1 \\ ORIF with circlage and \\ struts and plate. \\ Loose stem -B2 \\ Good bone stock \\ Yes- Revision with long stem, cemented or uncemented \\ No - Revision and augmentation of bone stock with allograft
}

\section{Type C}

Ignore implant, fix fracture, if necessary address implant after fracture healed.

We therefore decided to use a hybridization of various techniques to achieve what we felt could be the best possible result for our patient whose fracture would fall into the B1 type by the Vancouver

CONCLUSION: The fixation of periprosthetic fractures remains a challenge to even the most experienced orthopedic surgeon despite the common nature of the problem.

In physically and financially compromised patient, at times one has to think of some different modes of management. In this case we have used ender nail with fair result.

Long term follow up of this case is awaited.

We may follow up on this technique with more cases as and when required and hopefully present such cases to validate the statistical applicability of such technique.

\section{REFERENCES:}

1. Pape $\mathrm{H}$, Dvenger A., influence of different methods of femoral nailing in lung function of patients with multiple trauma. J trauma 1993. 35: 709.

2. Olerud S intramedullary reaming and nailing, its early effects on cortical bone vascularisation. or-thopaedics- 1986: 9 1204-08.

3. Kempf I, grossf, closed locked intramedullary JBJS(am) 1985: 67-A

4. Thorsen BO, Alho, interlocking intramedullary nailing in femoral shaft fracture, JBJS 1985 67-A

5. Wiss DA, fleming $\mathrm{CH}$, comminuted and rotationally unstable fracture of the femur treated with an interlocking nails.

6. Pankovich AM, closed ender nailing of femur shaft fracture JBJS 1979, 61-A.

7. Minns RJ, cambell J, biomechanical study of internal fixation of the tibial shaft, J Biomechanics 1997, 10-569-574.

8. Dobozi WR, Lawence Bg, flexible intramedullary nailing of subtrochanteric fracture femur, a multicentre analysis, clin. Ortho1988,212.

9. Moehring D, flexible intramedullary fixation of femoral fractures 1988, 227.

10. Muckle DS, ender nailing in femoral shaft fracture, injury 1981, 13. 
11. Pankovich AM, closed ender nailing of femoral shaft fracture JBJS 1979,61-A, 222-232.

12. Sirkin MS, femoral nailing without a fracture table, Clin ortho 1996, 332: 119-125.

13. Foulleras G, Ahlo A, locked intramedullary nailing of fracture femur and tibia injury 1990, 21 : 385-388.

14. Dankwardt $\mathrm{Mg}$, intramedullary nailing of femoral shaft after reaming of medullary cavity report on 6 years material 1973, 139: 155-156.

15. Clawson DK, closed intramedullary nailing of femur JBJS(am) 1971, 53-A: 681-691.

16. Lewin Pe, reduction exposure to the surgeon during intramedullary nailing JBJS (am) 1987 69A 761-765.

17. Miller ME,davis MI, prediction exposure and risk to operating room personnel during use of fluoroscopy guidance for selective orthopaedic procedure,JBJS (am) 1983, 65-A: 1-4.

18. Muller LP, radiation exposure to hands and thyroid of the surgeon during intramedullary nailing, injury 1998; 29(6) 461-468.

19. Krethek $\mathrm{C}$, undreamed intramedullary nailing of femoral shaft fracture, operative technique and early clinical experience with the standard locking optins; injury 1996, 27,(4) 233-254.

20. Krophl A undreamed intramedullary nailing of femoral fracture J. Trauma 1995,38, 717.

21. Klemn KW, interlocking nailing of complex fracture and tibia, cli Orthopaedics 1986,212,89100.

22. David, R. S. and Marvin E. S. The early history of arthroplasty in the United States. Clin. Orthop. Rel. Research 2000; 374: 55-89.

23. Ninan, T. M., Costa, M. L. and Krikler S. J. Classification of femoral periprosthetic fracture; Injury,Int. J. Care Injured2007; 38; 661-668.

24. Beals R. K., Tower S. S. Periprosthetic fractures of the Femur; Clin. Orthop. Relat. Res.1996; 327: 238-246.

25. Bethea, J. S., de Andrade, J. R., Fleming, L.L.et al. Proximal femoral fractures following total hip arthroplasty. Clin. Orthop. Rel. Res. 1982; 170: 95-106.

26. Brady, O. H., Kerry, R, Masri, B. A et al. The Vancouver Classification of periprosthetic fractures of the hip: a rational approach to treatment. Tech. Orthopaedi. 1999; 14(2): 107-14.

27. Cooke, P. H. and Newman, J. H. Fractures of the femur in relation to cemented hip prostheses; J. Bone Joint Surg.(Br). 1988; 70(3): 386-389.

28. Johansson, J. E, McBroom, R, Barrington T. W et al.Fracture of the ipsilateral femur in patients with total hip replacement: J. Bone Joint Surg. (Am) 1981; 63: 1435-1442.

29. Mont, M. A and Marr, D. C. Fractures of the ipsilateral femur after hip arthroplasty: a statistical analysis of outcome based on 487 patients. J. Arthroplasty 1994; 9: 511-519.

30. Whittaker, R. P., Sotos, L. N, Ralston, E. L. Fractures of the femur about endoprostheses. J. Trauma.1974; 14: 675-694.

31. Bassam, A. M, Dominic, R. M, Clive, P. D. Periprosthetic fractures evaluation and treatment. Clin. Orthop. Rel. Res.2004; 420: 80-95.

32. Tadross, T. S. F, Nanu, A. M, Buchanan, M. J et al. Dall-miles plating for periprosthetic B 1 fractures of the femur.J. Arthroplasty.2000; 15: 48-51.

33. Huaming, Xue, Yihui, Tu, Minwei, Cai et al. Locking compression plate and circlage band for type b1 periprosthetic femoral fractures: preliminary results at average 30-month follow-up. J. Arthroplasty (Article in Press). 
34. Dennis, M. G., Jordan, A. S., Kummer F. J. et al. Fixation of periprosthetic femoral shaft fractures occurring at the tip of the stem. A biomechanical study of 5 techniques. J. Arthroplasty. 2000; 15: 523-528.

35. Lever, J. O., Zdero, R, Nousiainen et al. The biomechanical analysis of three plating fixation systems for periprosthetic femoral fracture near the tip of a total hip arthroplasty. Mechanical Engineering Publications and Research 2010. Paper 5.

36. Richards, C. J., Duncan, C. P., Crawford RW. Cement-in-cement femoral revision for the treatment of highly selected Vancouver B2 periprosthetic fractures. J. Arthroplasty. 2011; 26: 335-338.

\section{AUTHORS:}

1. Bejoy E Jayan

2. Varunjikar M D.

3. Dnyaneshwar Kale

4. Suraj S. Mahadik

\section{PARTICULARS OF CONTRIBUTORS:}

1. Senior Resident, Department of Orthopaedics, Vikhe Patil Memorial Hospital.

2. Associate Professor, Department of Orthopaedics, Vikhe Patil Memorial Hospital.

3. Senior Resident, Department of Orthopaedics, Vikhe Patil Memorial Hospital.
4. Senior Resident, Department of Orthopaedics, Vikhe Patil Memorial Hospital.

\section{NAME ADDRESS EMAIL ID OF THE CORRESPONDING AUTHOR:}

Dr. Bejoy E. Jayan, e-403, Ganesh Plaza 2, Sector 1, Khanda Colony, New Panvel (w) - 410206. Email - bejoyjayan@gmail.com

Date of Submission: 07/10/2013. Date of Peer Review: 08/10/2013. Date of Acceptance: 11/11/2013. Date of Publishing: 14/11/2013 